\title{
Los cuidados de la salud en personas que viven con diabetes: enfoque etnográfico antropológico y perspectiva de género
}

\author{
Health care practices among people living with \\ diabetes: an anthropological, ethnographical \\ approach with a gender perspective
}

${ }^{1}$ Doctora en Antropología. Profesora Regular Adjunta, Universidad de Buenos

Aires. Investigadora, Instituto Interdisciplinario de Estudios de Género (IIEGE), Instituto de Investigaciones Gino Germani (IIGG), Universidad de Buenos Aires, Argentina. $\triangle$ iD
Ana Domínguez Mon ${ }^{1}$

RESUMEN La diabetes es una enfermedad que constituye un síndrome, cuyo crecimiento se registra con mayor velocidad en sociedades con condiciones de vida y de trabajo precarias y los cuidados cotidianos son fundamentales para controlar su progresión. El presente trabajo muestra el valor heurístico e interpretativo y la potencialidad explicativa del aporte del enfoque etnográfico y de la perspectiva de género para el análisis de las prácticas de cuidado en un grupo de adultos mayores que vive con diabetes tipo 2. El trabajo se llevó a cabo con un grupo de diabéticos y con profesionales de la salud en un centro de atención primaria de salud (CAPS) de José León Suárez, municipio de San Martín, provincia de Buenos Aires, en el período 2013-2016. Identificamos y analizamos acciones de cuidado (incluido el autocuidado) producto del conocimiento autorizado del grupo en articulación con los profesionales de salud del CAPS. Estas acciones individuales, grupales y colectivas conforman lógicas de cuidado que promueven el cuidado de sí. Esta propuesta metodológica se inscribe en la tradición del trabajo de campo colaborativo.

PALABRAS CLAVES Antropología Médica; Etnografía, Diabetes Mellitus Tipo 2; Género; Argentina.

\begin{abstract}
Diabetes, a disease that constitutes a syndrome, is growing more quickly in societies with precarious living and working conditions. Daily care practices are fundamental in preventing it from progressing. This work shows the heuristic and interpretative value and the explanatory potential of an ethnographic approach and a gender perspective in the analysis of care practices in a group of elder adults living with type 2 diabetes. The research was carried out with diabetes group participants and health professionals in a primary healthcare center in José León Suárez, municipality of San Martín, province of Buenos Aires, during the period 2013-2016. We identified and analyzed care activities (including self-care) that resulted from the authoritative knowledge of the group in connection with the healthcare center professionals. Such individual, group and collective actions generate care logics that promote care of oneself. The methodological proposal of this study is framed within the tradition of collaborative fieldwork.
\end{abstract}

KEY WORDS Medical Anthropology; Ethnography; Diabetes Mellitus, Type 2; Gender; Argentina. 


\section{INTRODUCCIÓN}

A especificidade do trabalho antropológico [...] em nada é incompatível com o trabalho conduzido por colegas de outras disciplinas sociais, particularmente quando, no exercício de sua atividade, articulam pesquisa empírica com interpretação de resultados. ${ }^{(1)}$

La teorización interdisciplinaria sobre el fenómeno género necesita los datos etnográficos para no perder la conexión con lo que realmente sucede en el mundo, y la antropología necesita la crítica feminista para desarrollar preguntas relevantes para entender el fenómeno género. ${ }^{(2)}$

Desde fines del siglo pasado, las afecciones crónicas o de larga duración se trasformaron en un problema destacado para las ciencias médicas y un desafío metodológico para las ciencias sociales ${ }^{(3,4)}$. A partir de la segunda mitad de siglo XX se promovieron investigaciones que permitieron avanzar en el mundo de los pacientes que viven con enfermedades crónicas, desde diversos abordajes teóricos y metodológicos: el interaccionismo simbólico, la teoría enraizada en los datos y los estudios inspirados en los aportes de la fenomenología de Paul Ricoeur. Todos ellos enfatizan sobre la necesidad de considerar el significado que adquiere la enfermedad crónica en el contexto de vida de las/os pacientes, entendiendo la particularidad de vivir con una afección no curable.

La diabetes ocupa además un lugar destacado entre los desafíos epidemiológicos del siglo XXI: por el aumento en la esperanza de vida de las poblaciones y porque su rápida expansión constituye una amenaza para los sistemas de salud, crecimiento de discapacidades a causa de eventos cardiovasculares y por los altos costos en diagnósticos, tratamientos e innovaciones terapéuticas. Existe evidencia de que las condiciones adversas de vida y de trabajo aceleran la aparición de enfermedades como la diabetes y la hipertensión, con mayor morbilidad y mortalidad en poblaciones socioeconómicamente vulnerables o en situación de pobreza ${ }^{(5,6)}$.

La diabetes se caracteriza por la imposibilidad del procesamiento adecuado de la glucemia a causa de la deficiente producción de insulina en el páncreas, generando lo que se conoce como "glucemia en sangre". En la actualidad existen taxonomías muy complejas, pero usualmente se reconocen dos tipos de diabetes: la diabetes tipo 1, ligada a la niñez y la adolescencia (también considerada una enfermedad autoinmune) y la tipo 2, propia sobre todo, aunque no exclusivamente, de adultos mayores, cuyo incremento se ha registrado en los últimos años en los países con mayores índices de pobreza ${ }^{(7)} y$, en los países centrales, en los grupos en los que se registra mayor desigualdad social, cultural y, por supuesto, económica ${ }^{(8)}$.

En Argentina, la diabetes es una enfermedad que alcanza a casi tres millones de enfer$\operatorname{mos}^{(9)}$. Es silente, dado que la gran mayoría de los afectados toman conocimiento luego de estudios de laboratorio, y está ligada a consumos de alimentos ricos en azúcares, carbohidratos, grasas y a estilos de vida sedentarios. En términos epidemiológicos, es un síndrome compuesto por un conjunto de factores que pueden aparecer también como patologías, como la obesidad.

Desde la tradición antropológica, la etnografía concentra tres acepciones: como enfoque, como método y como texto. El enfoque etnográfico constituye la piedra basal del trabajo de campo antropológico que busca reconocer variabilidad en las formas de vida de los conjuntos sociales a partir de sus propias miradas. El objetivo de esta producción de conocimiento es la comprensión y la explicación de fenómenos, en los términos de sus actores y con fines comparativos. Una interpretación comprensiva será la que se realiza a partir de los valores y las razones de las personas y no a partir de lo que el o la investigador/a considere que sean esas acciones. Es por ello que la antropología con base en el trabajo de campo ha desarrollado el método etnográfico, cuyo uso excede a la propia ciencia; su técnica primordial es la 
observación de la vida cotidiana de las personas bajo estudio. Esto presupone que un fenómeno como la enfermedad crónica, impacta no solo en el nivel somático, sino que afecta diversas dimensiones de la vida de las personas directa o indirectamente vinculadas a la diabetes. Observar la vida cotidiana de personas que viven con diabetes y comprender en sus propios términos el valor asignado a la enfermedad, habilita la interpretación y la comprensión de cómo ese fenómeno está articulado con otras dimensiones vitales usualmente no contempladas o subvaloradas en una consulta médica. Finalmente, los etnógrafos suelen plasmar sus trabajos en textos denominados etnografías, relatos que respetan las lógicas a partir de las cuales las propias personas han considerado sus afecciones. Podemos escribir sobre la diabetes como enfermedad, pero lo que no podremos hacer es reconocerla como padecimiento o sufrimiento si no es a partir de cómo las personas con diabetes viven esa afección. En este sentido, en el presente trabajo identificamos a las personas tal y como ellas se refieren a sí mismas en las entrevistas: diabéticos, registrados en bastardilla en el fragmento original y cuando se reproduzca en el texto.

El objetivo de este artículo es reconocer puentes entre el enfoque etnográfico y los estudios de género, puesto que ambos brindan elementos para comprender miradas o perspectivas usualmente negadas o poco exploradas en los trabajos tradicionales, particularmente, desde el campo de la antropología médica. Esta subdisciplina tomó de la antropología la crítica al universalismo propia del pensamiento médico occidental, sin lograr problematizarlo e incorporarlo como un sistema sociocultural más. Esta relación fue problematizada en la segunda posguerra del siglo $\mathrm{XX}$, cuando se incorporaron las teorías marxistas y del conflicto social, ya en su madurez, en los años sesenta. Hasta ese momento los análisis no lograban problematizar el conocimiento médico occidental, naturalizando las actividades de cuidado como intrínsecamente femeninas. Fue así como, desde la década de 1970, las diferentes teorías feministas dinamizaron críticamente el conocimiento producido desde la antropología ${ }^{(10)}$ e hicieron visibles las voces femeninas en los trabajos de campo, así como las propias en la academia. Sin embargo, entre antropología y feminismo siempre hubo una relación conflictiva: la antropología que se ocupó de la diferencia y la variabilidad no pudo dar cuenta en forma acabada de la articulación entre la variabilidad y la desigualdad en términos genéricos ${ }^{(11)}$, derivadas del origen colonial de esta disciplina. No es mi interés abarcar en esta introducción los encuentros y distanciamientos entre trabajo de campo etnográfico antropológico y los estudios de género, sobre todo a partir de los aportes de las teorías feministas. Podemos afirmar que a fines de la década de 1980, el aporte del enfoque etnográfico ha servido a los aportes teóricos de Pierre Bourdieu y Anthony Giddens, en la elaboración de teorías sobre la acción social y, simultáneamente, a la incorporación de las críticas provenientes del feminismo a la epistemología académica a través de la revisión del etnocentrismo occidental y sus derivaciones en el epistemocentrismo y androcentrismo en la ciencia moderna ${ }^{(10,12)}$. Nociones como resistencia, subalternidad y hegemonía fueron apropiadas y reelaboradas en las antropologías del sur. Los feminismos de la igualdad y de la diferencia fueron discutidos a partir de las miradas críticas de las teóricas latinoamericanas. La perspectiva de género se incorporó a las agendas de las agencias internacionales en la década de 1990 pero homologando género a mujeres, omitiendo en sus análisis que el género, al igual que la clase, es una categoría relacional ${ }^{(13)}$ y no una esencia relativa a los sexos, sino una construcción histórico-política.

En este trabajo propongo articular el enfoque etnográfico con la perspectiva de género, particularmente, con relación a personas mayores que viven con diabetes y que conformaron un grupo de pares, en un centro de salud público del primer nivel de atención en un municipio bonaerense.

Es común escuchar la afirmación de que "las mujeres cuidan porque está en su naturaleza". Aunque se contabilice la cantidad 
de personas que realizan actividades ligadas a los cuidados de la salud, que cuidan enfermos o dependientes y se compruebe que la mayoría son mujeres, no podría inferirse que sean más hábiles que los varones para esta actividad, sino que lo hacen en mayor proporción que los varones. Para comprender por qué, deberíamos indagar acerca de la división sexual del trabajo en las sociedades modernas.

Escogí los cuidados como eje de análisis porque suelen considerarse la piedra angular de las discusiones para el control de enfermedades no transmisibles o de larga duración, ya que la cronicidad remite a una situación irreversible de vida ${ }^{(14,15)}$ y no como momento en un proceso social y cultural mayor. Debido a que la categoría cuidados comprende una amplia gama de actividades destinadas al bienestar, entenderemos los cuidados de la salud como actividades prácticas concretas, particularmente, los cuidados de la salud que desarrollan las personas en función de brindar protección a quienes consideran en relación de dependencia (sea esta transitoria o permanente), o en situación de vulnerabilidad (también permanente o transitoria) y a toda aquella persona que lo requiera para el mantenimiento y sostenimiento de su vida. En los países del hemisferio norte se destacan los aportes antropológicos de Kleinman ${ }^{(16)}$ y Mol ${ }^{(17)}$. En Brasil y Argentina, María Epele ${ }^{(18)}$, Augusto Bonet ${ }^{(19)}$, Soraya Fleischer y Monica Franch ${ }^{(15)}$ y María Cristina Chardon en psicología ${ }^{(20)}$.

Los cuidados cotidianos de la salud son actividades que se realizan usualmente en el ámbito de la domesticidad. Consideraremos las acciones de cuidado como trabajo, sea este mercantilizado o no, y al cuidado no profesional como trabajo doméstico o trabajo privado no mercantilizado, según la bibliografía especializada ${ }^{(21)}$.

En general, las discusiones teóricas acerca de los cuidados se organizan alrededor de la idea de alteridad y de estatus de persona $^{(20)}$. ¿A quién o quiénes cuidar?, ¿quién debe/quiere hacerlo?, ¿cómo hacerlo?, comprenden gran parte de las discusiones teóricas y de políticas públicas en materia de cuida$\operatorname{dos}^{(22,23)}$. Podemos afirmar que los cuidados se han incorporado al análisis del proceso salud, enfermedad, atención de enfermedades de larga duración, sobre todo, desde la crisis del modelo curativo en la medicina moderna $^{(15,18,24)}$ que se origina, principalmente, a partir del tratamiento y cura de las enfermedades agudas durante la segunda mitad de siglo XIX.

La división sexual del trabajo constituye un criterio ordenador y controlador de las actividades productivas, así como de quiénes las llevan a cabo, sobre todo desde el orden capitalista industrial moderno. Ha reforzado social y moralmente el lugar subalterno femenino en tareas a las que se demanda "vocación" y "abnegación"(25), particularmente, en salud y educación.

Mi interés es mostrar el valor heurístico, interpretativo y explicativo de los aportes de la etnografía y de la perspectiva de género para el análisis de las prácticas de cuidado, particularmente, vinculadas a la diabetes en adultos mayores. Considero que ambos enfoques reconocen un punto de convergencia: que los significados sobre los fenómenos son diversos y variables, que los individuos disputan sentidos sobre estos según los capitales o recursos que dispongan en un campo determinado (recordando tanto a Bourdieu como a Giddens) en un momento histórico dado. Los resultados que obtuve son relativos a este grupo y en un momento particular que los relevé y analicé. Opondré perspectivas sobre la diabetes que remiten a marcos interpretativos en conflicto: el de la biomedicina diabetológica y el de la lógica práctica de cuidados de las personas que viven con diabetes. Desde el punto de vista metodológico, oponer miradas me permitió reconocer posibles puentes para un diálogo entre ambas lógicas. A tal fin, analizaré las relaciones de cuidado implicadas con la diabetes, basadas en el modelo del autocuidado diabetológico, cimentado en una lógica de elección consciente de acciones para lograr el control la enfermedad ${ }^{(17)}$. Esta perspectiva se expresa en los cuatro pilares tradicionales de la diabetología actual: la educación, la alimentación, la actividad física y el autocontrol. 
El autocontrol de la persona con diabetes consiste en que el propio paciente se haga una serie de análisis y reconocimientos sencillos. El objetivo del autocontrol de la diabetes es conseguir que tus niveles de azúcar en sangre estén lo más cerca posible del normal y mantenerlo así con la mayor frecuencia posible. ${ }^{(26)}$

Desde este marco interpretativo, estos cuatro pilares orientan y permiten, a través de un control médico, mantener o rectificar la terapéutica según la medicación disponible.

Por otro lado, las lógicas prácticas de cuidados son las acciones que las personas despliegan no siempre en forma consciente. Su identificación y análisis nos permiten reconocer dimensiones de la vida que escapan o no son reconocidas a través del autocuidado, también denominado por Annmarie Mol como "la lógica de la elección"(17), considerada práctica consciente e individual. Apelaremos al concepto cuidado de sí de Michel Foucault(27), reelaborado por Nora Muñoz Franco para el caso del cuidado en salud "como un concepto histórico que involucra la concepción y el referente que se tiene sobre sí y, en esa medida, sobre los otros"(28). Consideraremos al cuidado de sí en la relación de las personas con el propio cuerpo, con los otros y con el medio ambiente (entendiendo por nosotros como el contexto próximo). Estas nociones nos permiten reconocer lógicas de interpretación acerca de qué es vivir con diabetes, como en el caso del grupo indagado. Busco demostrar cómo este grupo ha promovido la reelaboración y resignificación de las experiencias individuales y grupales acerca de la diabetes, que habilitaron la emergencia de lógicas de cuidado desgenerizadas, es decir, no exclusivamente producidas por mujeres ${ }^{(24,29)}$.

\section{METODOLOGÍA DEL TRABAJO DE CAMPO}

Nuestro equipo está conformado por investigadoras del campo de las ciencias sociales, formadas en su mayoría en estudios de género y feminismo: antropólogas, sociólogas, comunicadoras sociales, filósofas, médicas generalistas, estudiantes de grado y de posgrado, abogadas y psicólogas, e integrantes del grupo de pares que vive con diabetes denominado Los Dulces de la Esperanza que será reconocido en este trabajo con la denominación abreviada de Los Dulces, y que se incorporó al equipo en 2014. Esta conformación interdisciplinaria permitió la combinación del trabajo académico con la experiencia territorial, habilitando la horizontalidad en la forma de trabajo intelectual y en la discusión de nuestros resultados.

Cada actividad realizada tuvo su proceso de explicación, discusión y consentimiento por parte del grupo Los Dulces: reuniones grupales, acompañamiento en actividades dentro y fuera del centro de atención primaria de salud (CAPS), así como la elaboración de dos videos en los que se volcó su experiencia con relación a los cuidados cotidianos de su salud como un grupo de pares. Esto se plasmó en un video documental Aprendiendo a cuidar(se) $)^{(30)}$, realizado en el año 2014, a través del cual tres miembros activos del grupo, con acuerdo de sus pares, relataron aspectos significativos acerca de lo que es para ellos vivir con la enfermedad.

Los datos que presentamos a continuación han sido registrados en el video de consulta pública, y que todos los participantes han consentido su realización. Contamos con la colaboración de un grupo de documentalistas de la Facultad de Ciencias Sociales de la Universidad de Buenos Aires (Fabián Fattore, Marcelo Burd y Martín Aratta) que se incorporaron al trabajo de campo en diálogo con Los Dulces en los días de reunión grupal. Para el registro de un lenguaje no sexista (puesto que en la lengua española los plurales son siempre masculinos) hemos escogido utilizar la letra " $x$ " para la inclusión de las minorías masculinas y solo referiremos en femenino cuando claramente el plural lo requiera. En el campo de la salud y sobre todo en el primer nivel de atención, la mayoría de Ixs usuarixs y de Ixs profesionales son mujeres. Inscribimos nuestro trabajo dentro de 
la metodología de etnografía colaborativa, propuesta dialógica e interlocutiva surgida en América Latina, particularmente, en proyectos interculturales: a través de la coautoría, en diálogo constante y consensuado con nuestrxs interlocutorxs en las producciones y en la generación de conocimiento ${ }^{(31)}$. Esta metodología surge como respuesta a las críticas a la autoridad etnográfica de investigadorxs académicxs y como resultado de las discusiones en el campo del activismo de los movimientos sociales indígenas, particularmente, por parte de feministas latinoamericanos a fines de siglo pasado y con gran desarrollo en las últimas décadas ${ }^{(32)}$.

\section{Los Dulces...}

El grupo de pares se creó el 20 de agosto de 2001 en un momento histórico particular de la Argentina, marcado por la crisis institucional y bancaria con bloqueo de cuentas y altos niveles de desocupación. Comenzaron nucleándose 14 participantes, actualmente lo conforman más de 30 personas: 22 mujeres y alrededor de 10 varones. La mayor parte vive en barrios céntricos de distintas localidades de la provincia de Buenos Aires: José León Suárez, Villa Ballester, Necochea, Caren, Hidalgo, Chilavert y Carcova. San Martín es un partido que históricamente fue industrial y que desde fines de la década de 1990 registró una pérdida de puestos de trabajo: se cerraron las fábricas y mucha gente quedó desempleada. En 2016 este proceso vuelve a registrarse. Los Dulces son adultos mayores de 50 años, la mayoría jubiladas/os. Salvo quienes poseen acceso a la seguridad social, la cobertura de salud proviene del Programa de Atención Médica Integral (PAMI) destinado a jubilados y pensionados. Los varones han desempeñado diversos trabajos: empleados públicos, cuentapropistas, obreros, un profesional y estudiante (un joven de 23 años). Las mujeres se reconocen como amas de casa y han sido empleadas domésticas, empleadas administrativas estatales, enfermeras, costureras en fábricas y algunas son cuentapropistas, o se dedican a la venta informal de productos elaborados (alimentos, manualidades) o la comercialización domiciliaria de utensilios de bazar. Dos son promotoras de salud que reciben una asignación municipal para realizar tareas de promoción. Los varones en situación de pobreza reciben subsidios del Estado a causa de alguna discapacidad (ceguera por diabetes), por situaciones familiares o socioeconómicas. Se destaca la participación de las mujeres en actividades barriales y dentro del CAPS. Del conjunto original quedan pocos miembros, por diversas razones: fallecimientos, mudanzas e imposibilidad para movilizarse por sus propios medios. De todos modos y en ritmo estable, el grupo ha crecido año a año.

\section{VIVIR CON DIABETES EN UN BARRIO DEL CONURBANO BONAERENSE DE SAN MARTÍN}

El municipio de San Martín es uno de los partidos con mayor población de la provincia de Buenos Aires, según el censo de 2010, posee 414.196 habitantes, de los cuales 198.094 son varones y 216.102 son mujeres, con un índice de masculinidad del 91,7 . El 9,1\% (37.850 personas) nacieron en el extranjero: un $44 \%$ de ellas es de origen paraguayo (16.717 personas) ${ }^{(7)}$. Está conformado por veintisiete localidades atravesadas de sureste a noroeste por el Ferrocarril Mitre. Sigue esta misma dirección la distribución de la población según condiciones geográficas desfavorables. La localidad de José León Suárez está ubicada al borde del río Reconquista, la segunda cuenca más contaminada del país. El Barrio Esperanza o Villa Corea, es un vecindario de casas bajas, en el que son habituales los episodios violentos. Entre Ixs usuarixs del CAPS 10 están Ixs primerxs habitantes, quienes recuerdan haber construido sus casas a mediados de siglo pasado: sin cloacas, ni red de agua potable, ni gas.

El centro de salud se estableció en el emplazamiento actual a comienzos de la década de 1980. Es una institución con visibilidad social, en un municipio que implementó 
el Programa de Diabetes de la Provincia de Buenos Aires (PRODIABA) en 1995, antes de que lo hiciera la provincia en 1996, lo que demuestra el dinamismo de esta institución para esa fecha. El grupo de Los Dulces, surgió con fines educativos vinculados a la diabetes ${ }^{(24,29,33)}$.

Con los años, esta práctica fue dando paso a otras instancias de trabajo, más vinculadas a la promoción y la prevención. Desde 2005, Los Dulces asisten invitados a los congresos de medicina general y jornadas institucionales del PRODIABA y de residentes de medicina general. En el CAPS se fueron sucediendo ofertas de apoyo psicológico, así como actividades de prevención secundaria para sus integrantes: talleres de actividades físicas, coordinados por un profesor de educación física, una instructora de gimnasia; de movimiento y postura corporales, coordinados por una kinesióloga; de cocina, coordinados por una nutricionista, así como las acciones promovidas por el grupo en articulación con Ixs profesionales de la institución. La creación y la dinámica grupal estuvieron atravesadas por la organización particular que se generó en el CAPS en los años noventa. Hasta 2016, sesionaban en asamblea, tenían una reunión semanal (los jueves de 9:30 a 11:30), con diversos tópicos. Todxs tienen y pueden pedir la palabra. En todas las observaciones y las entrevistas realizadas, Ixs integrantes manifestaron que produjeron cambios importantes para ellos y para la institución, han intervenido incluso en la elección del director del CAPS, han solicitado y obtenido el nombramiento de una podóloga en la institución. En 2009, elevaron una nota al Ministerio de Salud de la provincia de Buenos Aires por incumplimiento en la entrega de tiras reactivas, la nota fue firmada además por otros grupos del Gran Buenos Aires: José León Suárez, Hurlingham, Esteban Echeverría, Malvinas Argentinas, Moreno, Lomas de Zamora y 3 de Febrero. Tuvieron que realizar una demanda judicial y presentaron un amparo para la normalización de la entrega de las tiras reactivas. Consideran los logros en las demandas al Estado como resultado de su participación grupal y colectiva, con Ixs profesionales y lxs vecinos del barrio.
Esto ha favorecido el reconocimiento social de Los Dulces dentro de la institución y como interlocutores en espacios fuera del propio CAPS. De ser Ilamado "Alcatraz", en alusión a la cárcel estadounidense, el CAPS 10 hoy es referencia para quienes deseen formarse en medicina general, en trabajo social y en psicología en el primer nivel de atención. La planta profesional no ha variado sustancialmente en número (doce en 2001 y actualmente once), sino en estabilidad laboral: actualmente son solo seis los profesionales de planta.

\section{Aprendiendo a cuidar(se)}

Este es el título que dimos a uno de los audiovisuales que filmamos con Los Dulces y la participación de profesionales del CAPS, protagonistas en 2015 del segundo documental: El cuidado de Ixs cuidadores ${ }^{(34)}$.

En el primer film, Ixs integrantes fueron elegidxs en asamblea. Consensuamos qué momentos centrales de su vida deseábamos registrar y acordamos en escenificar prácticas relativas a los cuatro pilares diabetológicos: el control de glucemia; la toma de medicación; las comidas y las actividades físicas; la consulta médica y los encuentros grupales. La descripción y el análisis de estas acciones forman parte de trabajos discutidos en reuniones científicas y publicados por el equipo $^{(24,29)}$. Se buscó mostrar las acciones cotidianas para el autocuidado propuesto por el conocimiento diabetológico, cuya realización se cimienta en decisiones libres y autónomas. La medición diaria de la glucemia se realiza tantas veces en el día como lo requiera la toma de medicación y/o la administración de insulina. Si bien se desarrolla en la intimidad, forma parte de las actividades grupales, al inicio de las reuniones de los días jueves.

\section{La medición de la glucemia}

A medida que ingresan Ixs participantes un integrante toma una muestra de sangre y mide la glucemia de la mañana. En los inicios 
de la actividad (agosto de 2001) por la falta de recursos, la medición se hacía subdividiendo las tirillas reactivas que conseguían como donación de laboratorios o por PRODIABA. Son insumos imprescindibles para la medición de los niveles de glucemia en sangre y deben estar disponibles para todos los pacientes según lo establece la Ley 23753 de Problemática y Prevención de la Diabetes, sancionada y promulgada en 1989.

Hasta 2016, la gran mayoría de Los Dulces disponían de glucómetros y llegaban a la reunión con la información de los valores medidos en sus hogares. Es una instancia de control y también de contención: "Me dio 140 pero tuve una mala semana". No hay juicios de valor, los resultados se escuchan y se registran en una planilla. El énfasis está puesto en lograr que los valores negativos no alteren su bienestar. Es común la referencia a problemas de índole económica, así como a conflictos familiares en el aumento de las glucemias. Las mujeres refieren que las "emociones" y los "disgustos" son la principal razón. Este dato también fue registrado por Liliana Saslavski ${ }^{(35)}$ en su libro que plasma una investigación realizada en los años noventa.

\section{La medicación}

En diabetes, como en cualquier enfermedad de larga duración, la disponibilidad de recursos expresa calidades diferenciales en el cuidado cotidiano de la salud. Según la Ley $26914^{(36)}$, que modifica la Ley 23753 de 1989, todas las personas con diabetes deben disponer de la medicación obligatoria, en el subsistema de las obras sociales y medicina prepaga si son asalariados, así como en el subsistema público o en el PAMI. Desde 2014, con la reglamentación de la ley a través de la Resolución № 1156/2014, se amplía el espectro de medicamentos que deben ser provistos gratuitamente a los pacientes diabéticos. Hasta 2016, en el partido de San Martín, los 20 CAPS cubrían el $100 \%$ de los aparatos de medición rápida de glucemia, y las tirillas para control se entregaban regularmente. Por PRODIABA todo paciente que vive con diabetes recibe en forma gratuita insulina NPH (Neutral Protamine Hagedorn) e insulina corriente, algunos análogos ultralentos, metformina, glibenclamida y tiras reactivas. Hasta 2016, a través del programa Remediar del Ministerio de Salud de la Nación se entregaba metformina, glibenclamida, antihipertensivos y diuréticos. El municipio también recibía donaciones de medicamentos de laboratorios, según una comunicación personal con el coordinador de Enfermedades Crónicas de San Martín, en agosto de 2016. No siempre ocurría lo mismo con requerimientos especiales, como bombas de insulina o mayor consumo de tirillas. En ese caso, las indicaciones y el control médico eran indispensables para lograr su acceso gratuito. Si se negara el insumo, es importante que los enfermos sepan cómo y ante quién reclamar.

Annemarie $\mathrm{Mol}^{(17)}$ considera que el "cuidado" en diabetes a veces tiene el mismo efecto que la "cura". Disponer de una lapicera (pen) o de una bomba de infusión continua de insulina o de insulina de corrección rápida, no solo estimula una respuesta veloz del organismo, sino que produce, en términos simbólicos, algo semejante a la "cura". Esta eficacia simbólica no se produce solamente por el rápido efecto de la dosis, o por la ausencia de dolor ante el pinchazo. Me refiero a reconocerse parte de un sistema más justo, que repara y disminuye las desigualdades de quienes no podrían acceder a esos medicamentos y dispositivos a través del mercado. Estos recursos son parte del derecho que tienen por ley y su disponibilidad no debiera estar sujeta a las donaciones de los laboratorios. Un bien que circula como donación, no se considera un derecho sino una dádiva. Disponer de estas tecnologías se traduce en prácticas menos invasivas, más eficientes, decorosas y, por ende, menos estigmatizantes. En un segmento del film Aprendiendo a cuidar(se) ${ }^{(30)}$ Teo se administra insulina con una lapicera audible, indispensable por su ceguera. Ha participado como divulgador con conocimiento autorizado de su enfermedad en cuarto año de la carrera de ciencias de la salud en la Universidad Nacional de 
La Matanza. Ha explicado a los estudiantes cómo una persona ciega puede inyectarse eficientemente con esta mini inyección en formato lapicera.

\section{Las comidas y las actividades físicas}

En general los diabéticos tipo 2 presentan sobrepeso a causa de un escaso gasto energético. Por eso deben modificar sustancialmente su vida diaria: abandonar el consumo de azúcares, carbohidratos y grasas. Se sienten obligados a cambiar sus "gustos"(35), por alimentos que no les agradan o que consideran "con poco sabor". Es frecuente la referencia a lo que ya no pueden hacer, a veces como nostalgia y otras veces como reproche:

Yo no pienso lo que voy a comer en el día. Veo lo que tengo en la heladera y lo hago. No me gusta mucho la carne, es raro que coma carne... Yo me levanto me baño, y me tomo "el azúcar en ayunas" me pincho el dedo [se mide la glucemia] Tengo 126. [...] Tomo el desayuno, hago una colación antes del almuerzo o tomo una fruta. [Mis almuerzos] son así rápidos, hiervo verduras o fideos. Cuando vienen mis hijos es diferente, les hago lo que a ellos les guste. No soy de comer cosas que no podamos comer los diabéticos. Podemos comer de todo pero en poca cantidad. La gimnasia no me gusta pero caminar sí. Eso me gusta... [Mari, Aprendiendo a cuidar(se) $]^{(30)}$

Los talleres de cocina coordinados por la nutricionista son muy populares. Les resulta muy placentero incorporar recetas nuevas, pensar cómo reemplazar ingredientes y son momentos de júbilo grupal. Los Dulces aprendieron a cocinar "sano" aunque no siempre apliquen esas indicaciones al pie de la letra. Suelen relativizar las restricciones: "Nosotros podemos comer de todo pero en pequeñas cantidades" dice Mari en el film. Los talleres de cocina y las caminatas en un predio próximo al CAPS son actividades sostenidas en el tiempo, pero no constituyen transformaciones sustanciales de los hábitos alimentarios, ni podemos afirmar que efectivamente hayan incrementado el interés de varones y mujeres por los ejercicios físicos. Sin embargo, logran establecer rituales de encuentro, de intercambio y de bienestar que se vinculan estrechamente a lo que reconocemos como lógicas de cuidado, en tanto proceso que es sinuoso ${ }^{(17)}$ y recorre diversas instancias entre las cuales se entrecruzan las recomendaciones médicas, los gustos personales en los que participa la elección, pero sobre todo las posibilidades y los momentos de la vida de las personas.

Los lunes voy a cocina, a clases de cocina [en el instituto para discapacitados visuales]. Preparo algo, prepizzas, tartas, después almorzamos en grupos. Los jueves es la concurrencia al grupo de diabéticos. 9:30 es la reunión del grupo de diabéticos. A las 8:30 es la caminata con el grupo. [Teo, Aprendiendo a cuidar(se)] ${ }^{(30)}$

\section{Las actividades dentro del grupo: lógicas de cuidado}

Los varones que viven solos realizan tareas domésticas, aunque reciben asistencia de hijas, hermanas y/o vecinas. En el grupo, las mujeres retienen para sí muchas de las responsabilidades propias de la domesticidad: atienden consultas, Ilaman o visitan a sus compañerxs, buscan y reclaman insumos ante las autoridades estatales. En las actividades compartidas, se observa participación masculina en la contención a problemas personales, participan activamente y expresan sus sentimientos. Los varones elaboran comidas en los talleres de cocina y los han coordinado en varias ocasiones, en actividades lúdicas toman la iniciativa y sugieren actividades recreativas, participan de las reuniones barriales y comunitarias. Si bien persisten formas tradicionales de organización de las actividades basadas en la división sexual de trabajo doméstico, en el autocuidado 
personal e individual, comienza a reconocerse el valor de la red de pares, como sostén de la vida cotidiana de cada miembro.

Si pensamos en una red mayor, las instituciones de salud son la referencia obligada para los integrantes de Los Dulces:

Es todo un arte la relación entre el profesional y una persona que vive con diabetes. No es lo mismo un médico que te explica qué es lo que tenés que hacer y por qué lo tenés que hacer que el médico que te "baja línea". [Sebastián, Aprendiendo a cuidar(se)] $]^{(30)}$

Todxs recuerdan situaciones de maltrato en sus trayectorias institucionales, así como perciben cambios fundamentales en los procesos de cuidados a partir de su ingreso al CAPS 10, en donde los profesionales son "amigos" porque los sienten próximos, porque no los hostigan moralmente. Las actividades grupales integran un conjunto mayor de acciones, que consideramos como parte del cuidado de $s i^{i 37}$. Son consecuencia de un proceso institucional, en el cual se ven reconocidos en y por las prácticas solidarias que llevan a cabo y no como proceso individual o por ejercicio de autonomía personal ${ }^{(29)}$. Se expresan en la labor de escucha y acompañamiento que ellos mismos se prodigan a través de un proceso de trabajo grupal en relación con Ixs profesionales: no evaluando, no censurando, sino acompañando. En el film Aprendiendo a cuidar(se), el coordinador de Los Dulces destaca que "el profesional que no juzga, acompaña"(30). Este acompañamiento requiere, según nuestras observaciones, de una contención institucional. Los Dulces y Ixs profesionales del CAPS sostienen que es el principio fundamental para el tratamiento de una enfermedad de larga duración: el respeto del otro (persona, paciente, profesional). En el acto de reconocer al otro como semejante, están reconociéndose con capacidad de generar protección y en ese reconocimiento revalorizan esa actividad volviéndola valiosa para ellos mismos ${ }^{(16,38)}$ : "El acompañamiento respetuoso es una manera de cuidar" [Sebastián, Aprendiendo a cuidar(se)] ${ }^{(30)}$

\section{DISCUSIÓN}

La incorporación a un grupo de pares en el caso de la diabetes es un proceso por el cual cada integrante aprende de sus pares y es reconocidx como agente autorizado ${ }^{(39)}$. Saben de la enfermedad a partir de sus propias experiencias y de las de sus pares, trasladan ese conocimiento a la esfera pública, a otros grupos de pares, a profesionales y en el municipio. Este movimiento no se realiza sin conflictos como, por ejemplo, cuando defienden sus derechos. Este conocimiento autorizado posee una historicidad y les permite hablar de un nosotros, aun en situaciones de relato personal:

La constitución de un conocimiento autorizado es un proceso social contínuo que construye y refleja las relaciones de poder dentro de una comunidad de prácticas [...]. Esto lo hace de tal manera que todos los participantes pasan a ver el orden social actual como un orden natural; así es como son (obviamente) las cosas. [Traducción del original: The constitution of authoritative knowledge is an ongoing social process that both builds and reflects power relationships within a community of practice [...]. It does this in such a way that all participants come to see the current social order as a natural order; that is the way things (obviously) are.] ${ }^{(39)}$

Algo similar ocurre cuando recuerdan el diagnóstico, ya lejano en el tiempo, y lo reconstruyen a partir de cómo piensan actualmente su tratamiento, no como parte de una conmoción biográfica, sino resignificado a partir de la experiencia actual:

Cuando te diagnostican la enfermedad... con la bajada de línea de la biomedicina, te genera una imposición de límites... [pero] en realidad te coarta la libertad. La información es muy útil, pero por sí sola no basta. [...] Es difícil porque con todos los quilombos que tiene la vida 
cotidiana, la salud pasa a un segundo plano. Cuando uno está en un grupo, la enfermedad la tenés en agenda. [Sebastián, Aprendiendo a cuidar(se) $]^{(30)}$

Evocan emotivamente alguna situación vivida en forma conflictiva. El desenlace de la enfermedad es reinterpretado en términos del contexto en el cual recibieron el diagnóstico o como desencadenante de él: crisis familiares, laborales, personales, etc.

Me dijeron los médicos que la diabetes era una enfermedad incurable pero silenciosa. Pensé que era como el cáncer [...] Después lloré, lloré todo el día y toda la noche hasta que dije "Mari ponete las pilas y pensá que te tenés que cuidar y seguir para adelante". Y fui al centro que me enteré que había un grupo de diabéticos. Yo ya sé que cuando estoy mal o estoy nerviosa, me sube el azúcar. [Mari, Aprendiendo a cuidar(se) $]^{(30)}$

Para el modelo médico diabetológico, el autocuidado es el resultado de la elección libre, individual y consciente de los pacientes, como expresión de la autonomía de quienes sabiéndose enfermos actúan para disminuir los factores de riesgo. Los Dulces discuten prácticamente con esta lógica de la elección consciente y libre. A partir de las acciones observadas y en las entrevistas reconocen las limitaciones. Saben que por más que sigan las indicaciones, la enfermedad puede progresar y eso los desalienta al mismo tiempo que los enfrenta a la incertidumbre de las complicaciones: retinopatías, neuropatías, entre las más temidas. Discuten y se enfrentan con las advertencias morales médicas cuando son esgrimidas como amenaza o como "castigo" por las transgresiones. Saben que la posibilidad de elegir llevar a cabo la indicación, está fuertemente condicionada por las condiciones de vida que tienen. De este modo, la insulinización se percibe como el castigo a un defectuoso cumplimiento de las indicaciones médicas ("Me dijo, si seguís así vas a terminar siendo insulinodependiente"). Este "temor", muchas veces mencionado en las reuniones, opera también como disciplinador, pero solo en forma momentánea. "No se puede vivir con miedo" afirma Sebastián en el film, en su doble condición de diabético y de profesional de la salud. El miedo entorpece la búsqueda de ayuda; algunos miembros, particularmente los varones, han demorado en iniciar el tratamiento por el temor que les produjo el diagnóstico, al no sentir dolor, consideraron que las advertencias médicas eran infundadas. En otros casos este temor aceleró procesos que derivaron en complicaciones que se manifiestan con mayor frecuencia, aunque no exclusivamente en varones. Desde el campo de la antropología, sabemos que los consumos y los gustos han sido culturalmente y económicamente construidos en articulación con valores de clase. El desarrollo del capitalismo en las sociedades modernas ha demostrado que el consumo de azúcar, carbohidratos y comidas rápidas ricas en grasas saturadas conduce a la malnutrición, el sobrepeso y la obesidad mórbida $^{(39,40)}$, todas enfermedades evidencian condiciones de vida precarias.

Desde las ciencias sociales y particularmente desde la etnografía, buscamos problematizar el sentido común establecido: "los pacientes no se hacen cargo de lo que les pasa", "mienten", "son transgresores". No buscamos oponer el sentido común médico a la agencia de las personas que viven con diabetes, quienes muchas veces ponen al límite su salud física. Sabemos que los sentidos de cuidado, desde la perspectiva de los propios protagonistas, evidencian agentes que pueden adherir como rebelarse frente a las indicaciones $\mathrm{y}$, al mismo tiempo, no se reconocen como víctimas. En el caso estudiado los conocimientos autorizados en y a partir de acciones grupales y colectivas, adquieren otro cariz; surgen de situaciones de clase, de género y étnicas subalternas. Lxs paraguayxs residentes durante más de dos décadas en el municipio de San Martín, con hijos y nietos argentinos, valoran la atención gratuita, contrastándola con la que reciben sus familiares en Paraguay. En este grupo son las mujeres, sobre todo, quienes vivieron situaciones de alta desigualdad, abuso sexual en 
Ios trabajos, maltrato marital y familiar, y que se redefinieron en su condición de adultas mayores.

En la bibliografía sobre cuidados de la salud, es común la referencia a la demora de los varones para realizar la primera consulta y, luego del diagnóstico, para iniciar el tratamiento. Esto revelaría el escaso valor que los varones ${ }^{(28)}$ asignan a la "prevención", supuestamente por ausencia de valores vinculados a los cuidados del cuerpo, más allá de los necesarios para trabajar. En cambio, se resalta la consulta temprana femenina, así como su mayor apego a las recomendaciones médicas. En el CAPS 10 los varones que cumplen con las indicaciones suelen disponer de redes de cuidado articuladas por mujeres (personal de salud, familiares y vecinas) que los asisten en la vida cotidiana, no es menos cierto que tanto para las mujeres como para los varones que están dentro de redes protectoras, las acciones de cuidado se reconocen como efectivas ${ }^{(42)}$.

Sin embargo, desde enero de 2015 al 1 de septiembre 2016, en el CAPS 10 se atendieron 373 personas con diabetes, 186 mujeres y 187 hombres que realizaron en total 1.835 consultas con distintos profesionales. ¿Acaso esta paridad obedezca a un reconocimiento de la labor institucional del CAPS en el barrio?

En el caso de este grupo de adultxs mayorxs, hemos identificado cambios en sus trayectorias de vida: lo que en la juventud y la adultez fue motivo de postergación, por privaciones económicas, por temores personales o imposiciones familiares, se torna logro personal, sobre todo, en las mujeres en esta etapa vital: "antes me callaba"; "antes acataba". Las mujeres que en su juventud aceptaron órdenes patriarcales, como cuándo y con quién casarse, cómo llevar la casa, cómo criar los hijos, pasados los cuarenta años producen cambios vitales importantes. Se separan de sus compañeros/maridos, o viven en la misma casa sin cohabitación conyugal, trabajan siendo jubiladas por razones estrictamente económicas o por interés personal; colaboran en instituciones o desarrollan formas de trabajo comunitario (la mayor parte de las promotoras de salud son mujeres), ingresan a los bachilleratos de adultos o a los grupos de la tercera edad. Han producido cambios significativos en sus vidas. La enfermedad es uno de los motivos del cambio, pero no el único ${ }^{(43)}$.

En este grupo de adultos mayores los varones aprendieron a cocinar, no solo para sí, sino para el grupo; prueban alimentos que rechazaban cuando sus esposas o parejas los preparaban, expresan emociones en las reuniones grupales, preparan dramatizaciones y evocan experiencias de vida con hijos y nietos. Estos adultos tan apegados al trabajo, a las obligaciones y a las jerarquías durante gran parte de sus vidas, se reconocen como parte de un colectivo. Han finalizado la etapa productiva, algunos han perdido a sus compañerxs de vida, otrxs han decidido separarse o divorciarse. Los estudios sobre adultos mayores y diabetes demuestran que si bien la diabetes tiene mayor prevalencia en las mujeres adultas, los varones afectados fallecen antes que las mujeres ${ }^{(44)}$. Existen trabajos que destacan el papel de las mujeres en las redes sociales por contrapartida a la escasa participación de los varones. Desde comienzos de siglo XXI se ha enfatizado en el valor de los grupos de pares ${ }^{(45)}$ como estrategia protectora, particularmente, en las enfermedades de larga duración y que por ahora no tienen cura.

\section{APERTURAS A MODO DE CIERRE}

El enfoque etnográfico y la perspectiva de género son estrategias metodológicas ricas para la comprensión de los cuidados de la salud en personas que viven con diabetes. La bibliografía disponible para enfermedades crónicas desde el último tercio del siglo pasado ha demostrado que existe una distancia muy grande entre la enfermedad y el sufrimiento y que este último puede afectar a las personas deteriorando su salud y provocando tempranamente la muerte. Acordamos con los estudios realizados por feministas sobre los cuidados cotidianos ${ }^{(38)}$, que la desigualdad en la división sexual de las actividades 
cotidianas de cuidado genera malestar y sufrimiento en las personas que cuidan y que estas son predominantemente mujeres. Los grupos de pares de adultos mayores que viven con diabetes, como es el caso de Los Dulces, evidencian transformaciones en los roles tradicionales basados en la división sexual del trabajo. No podemos afirmar que sean transformaciones producto de cuidados basados en una lógica de elección libre, sino en una lógica de cuidados producto de la dinámica grupal e institucional registrada y relativa a los contextos históricos institucionales que acompañaron hasta el momento su funcionamiento. Afirmamos que reconocer, pensar y realizar acciones, no adscribiéndolas a ningún sexo determinado, descomprime y vacía lugares de poder jerárquico, a la vez que otorga mayor libertad y creatividad en su ejecución. Esto es un trabajo lento, una predisposición al reconocimiento del error y al ejercicio de aceptación para recibir y producir cuidados. Acompañamiento respetuoso es cuidado, tanto como la asistencia y la responsabilidad del otro como semejante. Las escenas de acompañamiento de varones a otrxs miembros del grupo se observan y rara vez se mencionan en las entrevistas o en la donación de medicamentos o insumos no utilizados. Esto modifica la percepción de quién es el otro para el que se ejecuta la acción de cuidar.

En el trabajo de campo etnográfico, la posibilidad de identificar puntos de vista y explicar relaciones y disputas no se logró a través de las entrevistas, sino de los gestos, las miradas, los silencios. Cito a continuación una nota de campo tomada en 2016 en el CAPS:

Proyectamos "El cuidado de Ixs cuidadorxs", durante la última hora de reunión de Los Dulces. Inicialmente seríamos unas quince personas. Observé que fue ingresando gente y no parecía que era por el film. Cuando finalizó el video comprobé que el salón de usos múltiples estaba lleno: profesionales que conozco de hace tres años y vecinos que aún no conozco. No se habían enterado de la proyección, iban a una asamblea.
Si bien los primeros intercambios tenían que ver con el video, reparé que en realidad comentaban sus propias historias al referirse a las de los profesionales del film. Se sucedían intercambios sobre las responsabilidades médicas y la relación con los pacientes y con las autoridades sanitarias. Buscaban conceptualizar ideas acerca del cuidado, a partir de sus formas de trabajo: la escucha, la participación y el compromiso con el sufrimiento social ¿Cómo medirlo en prestaciones? ¿Cómo traducir la calidad en números? Detrás de las personas que hablaban, yo reconocía profesionales comprometidos, que evidencian respeto hacia las personas, y practican la lógica de los cuidados a partir del reconocimiento de la situación del sufrimiento social de sus pacientes. A través de acciones concretas: cuando salen a buscar a un paciente que hace meses que no va a la consulta, cuando organizan estrategias deportivas o lúdicas para que los pibes del barrio se acerquen, confíen en ellos; que las madres hablen y ellos entiendan, aprendan qué les pasa... Sentados en un salón repleto de gente con ganas de hablar y de escucharse, en un centro de salud ubicado en una zona de alta conflictividad social, estaban tratando de pensar su práctica supuestamente a propósito del film. Mientras se producían los intercambios sobre el valor de la solidaridad con la gente (presente) y de la gente con sus profesionales, delante de mí, un miembro de Los Dulces se acercó al coordinador del grupo y en voz baja susurró algo que no alcancé a escuchar por las voces de varias decenas de personas que desbordaban el salón. Le entregó dos cajas de insulina NPH y un glucómetro. Me mira el coordinador y me dice: "¿Querés un ejemplo de solidaridad? Esto ya está comprometido para un pibe de 16 [años]". (Nota de campo, 2016)

Si lo pensamos en términos metodológicos, la perspectiva de género en la observación de acciones cotidianas, nos permite 
dirigir teóricamente la mirada a aspectos o dimensiones ocultas o imperceptibles para la mirada no entrenada. No podemos afirmar que no perduren estereotipos de género en las actividades cotidianas de cuidado entre mujeres y varones. Pero hemos percibido cambios que tienen que ver con la dinámica grupal y, pensamos, con el momento vital que atraviesan. El trabajo etnográfico y la perspectiva de género han permitido comprender, desde la lógica de cuidados de Ixs interlocutorxs, el tipo de conocimiento autorizado que poseen y no solo desde la enfermedad como límite, sino como expresión de agencia, incorporada en el cuidado de sí a partir de prácticas grupales de cuidado. Los gestos que aprendí a reconocer, son los que ellxs me habilitaron a mirar en los términos en los que ellos desean ser reconocidos.

El enfoque etnográfico, al igual que la perspectiva de género, brinda carnadura a gestos, nos permite comprender relaciones complejas a partir de interacciones simples. La desgenerización de las prácticas es una tarea lenta y muy conflictiva que puede percibirse en pequeñas acciones. El trabajo grupal entre pares para generar el cuidado de sí requiere, sobre todo, ceder posiciones cómodas de dominio que muchas veces generan sufrimiento: los varones que no logran afrontar la diabetes hasta que perciben las complicaciones o que acuden a las consultas con mayor deterioro que las mujeres. Los mandatos de género en términos de cuidado no son fácilmente modificables en sociedades en las que persiste la división sexual del trabajo o, por lo menos, se lo naturaliza. Mientras las tareas de cuidado sean consideradas primordialmente femeninas, que las instituciones en el primer nivel de atención sean sostenidas por mujeres en condiciones de subalternidad, la desgenerización de las prácticas de cuidado de la salud será poco visible.

\section{AGRADECIMIENTOS}

Este trabajo fue posible gracias al financiamiento del proyecto UBACyT 20020130100636BA "Cuidados, cuidadoras/es y derechos en personas con enfermedades no transmisibles", convocatoria 2014-2017.

\section{REFERENCIAS BIBLIOGRÁFICAS}

1. Cardoso Oliveira R. O trabalho do antropólogo. São Paulo: UNESP, Paralelo 15; 2000.

2. Thurén BM. La crítica feminista y la antropología: una relación incómoda y fructífera. Ankulegi. 2008;(12):97-114.

3. Mendes Diz AM. Las enfermedades crónicas: Un nuevo paradigma de abordaje desde lo médico y lo social. In: Domínguez Mon A. (comp.). Agencia y cuidados en personas que viven con enfermedades crónicas no transmisibles (Documento de trabajo $N^{\circ} 60$ ). Buenos Aires: Instituto de Investigaciones Gino Germani, Facultad de Ciencias Sociales, Universidad de Buenos Aires; 2012.

4. Castellanos MEP, Trad LAB, Jorge MSB, Leitão IMTA. Cronicidade: experiencia de adoecimento e cuidado sob a ótica das ciências sociais. Fortaleza: EdUECE, 2015.

5. Salas J. La desigualdad social, el mosquito que inocula enfermedades crónicas. El País [Internet]. 24 feb 2016 [citado 24 feb 2016]: Sec. Ciencia. Disponible en: https://tinyurl.com/j9cd85j.

6. Marmot M. The health gap: the challenge of an unequal world. The Lancet. 2015;386:2442-2444.

7. Pérez S, Perner S. Prácticas alimentarias y actividades físicas en contextos de desigualdad. In: Domínguez Mon A, Schwarz P. (comp.). Redes de cuidado, autocuidado y desigualdad en salud: personas que viven con enfermedades de larga duración (Documentos de Trabajo No75). Buenos Aires: Instituto de Investigaciones Gino Germani, Facultad de Ciencias Sociales, Universidad de Buenos Aires; 2015.

8. Organización Mundial de la Salud. Informe Mundial sobre la Diabetes [Internet]. OMS; 2016 [citado 10 may 2016]. Disponible en: https:// tinyurl.com/y8n3hy9z.

9. Czubaj F. En la Argentina, uno de cada 10 adultos sufre de diabetes. La Nación [Internet]. 7 abr 2016 [citado 10 may 2016]. Sec. Vida y Ocio. Disponible en: https://tinyurl.com/yd4wdg3u. 
10. Moore C. El proceso de mediación: Métodos prácticos para la resolución de conflictos. Barcelona: Ediciones Granica; 1995.

11. Strathern M. Una relación dificultosa: el caso del feminismo y la antropología. Feminaria. 1990;3(6):1-9.

12. Harding S. ¿Existe una metodología feminista? In: Adán C, Villamarín CA, Sedeño EP, Romero MJ. Feminismo y conocimiento. La Coruña: Espiral Maior; 2006.

13. Stolcke V. ¿Es el sexo para el género lo que la raza para la etnicidad... Y la naturaleza para la sociedad? Política y Cultura. 2000;(14):25-60.

14. Passerino L. ¿Qué es la cronicidad? Experiencia y subjetividad en el tratamiento discursivo del cáncer. Simposio Nacional del Padecimiento a la Agencia Social en Salud Colectiva: Personas que viven con enfermedades crónicas y vida cotidiana; 25-26 oct 2012; San Carlos de Bariloche, Argentina.

15. Fleischer S, Franch M. Uma dor que não passa: aportes teórico-metodológicos de uma antropologia das doenças compridas. Politica \& Trabalho. 2015;(42):13-28.

16. Kleinman A, Van der Geest S. 'Care' in health care: remaking the moral world of medicine. Medische Antropologie. 2009;21(1):159-168.

17. Mol A. The logic of care: Health and the problem of patient choice. Oxon: Taylor \& Francis; 2008.

18. Epele M. Padecer, cuidar y tratar: estudios socio-antropológicos sobre consumo problemático de drogas. Buenos Aires: Antropofabia; 2013.

19. Bonet OAR, Tavares F, Gomes R. O cuidado como metáfora nas redes da prática terapêutica. In: Pinheiro R, Mattos R. (eds.). Razões públicas para a integralidade em saúde: o cuidado como valor. Rio de Janeiro: CEPESC-IMS, UERJ, ABRASCO; 2007.

20. Chardon MC. Representaciones sociales del cuidado: entre las prácticas y la noción de alteridad. Arquivos Brasileiros de Psicologia. 2008;60(2):10-19.

21. Topalov C. La urbanización capitalista. México: Edicol; 1979.

22. Pautassi LC. El cuidado como cuestión social desde un enfoque de derechos (Serie Mujer y Desarrollo). Santiago de Chile: Naciones Unidas; 2007.
23. Pautassi LC, Zibechi C. Las fronteras del cuidado: agenda, derechos e infraestructura. Buenos Aires: Biblos, ELA; 2013.

24. Domínguez Mon AB. Cuidados de sí y relaciones de género: trabajo en red en un grupo de personas que viven con diabetes (PVD) en un centro de atención primaria de la salud (CAP) en José León Suárez, Argentina. Política \& Trabalho. 2015;(42):133-154.

25. Martin AL. Parir, cuidar y asistir: El trabajo de las parteras y enfermeras en Buenos Aires (1877-1955). [Tesis de doctorado]. Buenos Aires: Facultad de Filosofía y Letras, Universidad de Buenos Aires; 2014.

26. Countour Diabetes Solutions [Internet]. Ascensia Diabetes Care; 2010 [citado jun 2014]. Disponible en: https://goo.gl/QUNFYH.

27. Foucault M. Hermenéutica del sujeto. Buenos Aires: FCE; 2006.

28. Muñoz Franco NE. Aprendizajes de género y cuidado de sí en la salud masculina: entre lo universal y lo específico. Psicología, Conocimiento y Sociedad. 2012;2(2):6-26.

29. Domínguez Mon AB. Prácticas cotidianas de (auto) cuidados en red por parte de un grupo de pacientes con diabetes (DBT). In: Domínguez Mon A, Schwarz P. (comp.). Redes de cuidado, autocuidado y desigualdad en salud: personas que viven con enfermedades de larga duración (Documentos de Trabajo N075). Buenos Aires: Instituto de Investigaciones Gino Germani, Facultad de Ciencias Sociales, Universidad de Buenos Aires; 2015.

30. Instituto de Investigaciones Gino Germani. Aprendiendo a cuidar(se). [Video]. 2014 [citado 10 may 2016]. Disponible en: https://goo.gl/6hLy5M.

31. Rappaport J. Más allá de la observación participante: la etnografía colaborativa como innovación teórica. In: Leyva X, Hernández RA, Alonso J, Báez M, Kölher A, Escobar A, Krotz E, Campos MD, Restrepo E, Flores JA, Reartes D. Conocimientos y prácticas políticas: reflexiones desde nuestras prácticas de conocimiento situado (Tomo II). Chiapas: CIESAS, UNICACH, PDTG-UNMSM; 2011.

32. Hernández Castillo RA. Hacia una antropología socialmente comprometida desde una perspectiva dialógica y feminista. In: Leyva X, Hernández RA, Alonso J, Báez M, Kölher A, Escobar A, Krotz E, Campos MD, Restrepo E, Flores JA, Reartes D. Conocimientos y prácticas políticas: reflexiones desde nuestras prácticas de conocimiento situado (Tomo I). Chiapas: CIESAS, UNICACH, PDTG-UNMSM; 2011. 
33. Peresin S. Valoración desde la perspectiva de los usuarios, de un dispositivo de psicoeducación en diabetes elaborado e implementado en el Centro de Salud N ${ }^{\circ} 10$ "Barrio Esperanza" (San Martín - Bs. As.). [Tesis de Maestría]. Rosario: Instituto de la Salud "Juan Lazarte", Centro de Estudios Interdisciplinarios, Universidad Nacional de Rosario; 2011.

34. Instituto de Investigaciones Gino Germani. "El cuidado de los cuidadorxs" [Audio]. 2015 [citado 10 may 2016]. Disponible en: https://goo. gl/ohBXUJ.

35. Saslavski L. ¿Por qué no se cura (todavía) la diabetes? Buenos Aires: Antropofagia; 2007.

36. República Argentina. Ley 26914 [Internet]. 2013 [citado 10 may 2016]. Disponible en: https:// tinyurl.com/y6uqrnal.

37. Muñoz Franco NE. Reflexiones sobre el cuidado de sí como categoría de análisis en salud. Salud Colectiva. 2009;5(3):391-401.

38. Benhabib S. Una revisión del debate sobre las mujeres y la teoría moral. Isegoría. 1992;(16):37-63.

39. Jordan B. Authoritative knowledge and its construction. In: Davis Floyd R, Sargent C. Childbirth and authoritative knowledge: cross-cultural perspectives. California: University of California Press; 1997.

40. Gracia-Arnaiz M. Comer bien, comer mal: la medicalización del comportamiento. Salud Pública de México. 2007;49(3):236-242.

41. Aguirre P. Ricos flacos y pobres gordos. Buenos Aires: Capital Intelectual; 2004.

42. Fuentevilla GC, Ríos RM, Gordillo G. Diabetes Mellitus II: la importancia de las redes de apoyo como soporte al padecimiento. Población y Salud en Mesoamérica. 2011;9(1):2-22.

43. Neves E. Viver com (e apesar de) a doença: apontamentos sobre a experiência sobre adoecimentos crônico entre diabéticos da ADJP/PB Brasil. Política \& Trabalho. 2015;(42):11-131.

44. Trujillo Olivera LE, Nazar-Beutelspacher A, Salvatierra-Izaba B. Autocuidado de diabetes: una mirada con perspectiva de género. Estudios Demográficos y Urbanos. 2011;2(3):639-669.

45. Sapag JC, Lange I, Campos S, Piette JD. Innovative care and self-care strategies for people with chronic diseases in Latin America. Revista Panamericana de Salud Pública. 2010;27(1):1-9.

\section{FORMA DE CITAR}

Domínguez Mon A. Los cuidados de la salud en personas que viven con diabetes: enfoque etnográfico antropológico y perspectiva de género. Salud Colectiva. 2017;13(3):375-390. doi: 10.18294/sc.2017.1156.

Recibido: 26 de septiembre de 2016 | Versión final: 20 de marzo de 2017 | Aprobado: 4 de abril de 2017

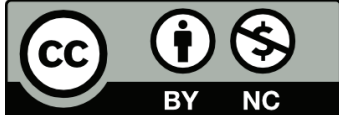

Este obra está bajo una licencia de Creative Commons Reconocimiento-NoComercial 4.0 Internacional. Reconocimiento - Permite copiar, distribuir y comunicar públicamente la obra. A cambio, se debe reconocer y citar al autor original. No Comercial - Esta obra no puede ser utilizada con finalidades comerciales, a menos que se obtenga el permiso. 